\title{
Control and Stabilization of A Rotating Flexible Structure $* \dagger$
}

\section{ÖMER MORGÜL}

Key Words-Distributed parameter systems; partial differential equations; boundary-value problems; stability; Lyapunov methods.

\begin{abstract}
We consider a flexible beam clamped to a rigid base at one end and free at the other end. We assume that the rigid base rotates with a constant angular velocity and that the motion of the flexible beam takes place on a plane. To suppress the beam vibrations, we propose dynamic control laws for boundary control force and torque, both applied to the free end of the beam. We show that, under some conditions, one of which is the strict positive realness of the actuator transfer functions which generate the boundary control force and torque, the beam vibrations asymptotically decay to zero if the rigid base angular frequency is sufficiently small. Moreover, if the transfer functions are proper but not strictly proper, we show that the decay is exponential. We also give a bound on the constant angular velocity above which the system becomes unstable.
\end{abstract}

\section{Introduction}

MANY MECHANICAL SYSTEMS, such as spacecraft with flexible appendages or robot arms with flexible links, can be modeled as coupled elastic and rigid parts. Many future space applications rely on lightweight materials and high performance control systems for high precision pointing, tracking, etc. To achieve high precision demands for such systems, one has to take the dynamic effect of flexible parts into account. Thus, over the last decade there has been a growing interest in obtaining new methods for the design, analysis and control of the systems which have flexible parts.

Stability of systems which have elastic parts, particularly flexible space structures, has been studied in the past. More recently, Baillieul and Levi (1987) considered the dynamics and the stability of a rotating flexible structure from a Lagrangian point of view and studied the asymptotic dynamics.

In recent years, the boundary control of flexible systems (i.e. controls applied to the boundaries of the flexible parts as opposed to the controls distributed over the flexible parts) has become an important research area. This idea was first applied to systems described by the wave equation (e.g. strings), Chen (1979) and recently has been extended to the Euler-Bernoulli beam equation, Chen et al. (1987). In particular, in Chen et al. (1987), it has been proven that, in a cantilever beam, a single actuator applied at the free end of the beam is sufficient to uniformly stabilize the beam deflections. Recently, the boundary control techniques has

* Received 3 November 1992; received in final form: 12 March 1993. This paper was not presented at any IFAC meeting. This paper was recommended for publication in revised form by Associate Editor R. Curtain under the direction of Editor Huibert Kwakernaak.Corresponding author O Morgül. Tel. +90426640 00/15 29; Fax +904266 41 27; E-mail: morgul@trbilun.bitnet.

$\dagger$ This research has been supported by TÜBITAK, the Scientific and Technical Research Council of Turkey under the grant TBAG-1116.

$\ddagger$ Department of Electrical and Electronics Engineering, Bilkent University, Bilkent, 06533, Ankara, Turkey. been applied to the stabilization of rotating fiexible structures, see Morgül (1990, 1991).

In this paper, we study the motion of a flexible beam, which may model a flexible robot arm, clamped to a rigid body at one end and free at the other end. We assume that the center of mass of the rigid body is fixed in an inertial frame and that the rigid body rotates in that frame with constant angular velocity. We also assume that the motion takes place on a plane. To suppress the beam vibrations, we apply a dynamic boundary control at the free end of the beam. With some assumptions on the actuator which generates this boundary control, we prove that the beam vibrations decay asymptotically to zero. We give a characterization of the greatest angular velocity above which the rotating flexible beam becomes unstable.

\section{Equations of motion}

We consider a flexible structure which consists of a rigid body with a flexible beam clamped to it at one end, the other end of the beam is free. We assume that the center of mass of the rigid body is fixed in an inertial frame and that the whole structure performs planar motion with a constant angular frequency $\Omega$. We assume that at rest the flexible beam is straight. Let $P$ be a point of the beam whose distance from the clamped end at rest is $x$ and let $u$ be the displacement of $P$ when the beam vibrates.

Neglecting gravitation, surface loads and the rotatory inertia of the beam cross-sections and using the EulerBernoulli beam model, the equations of motion of the system are: for $t \geq 0$

$$
\begin{gathered}
E l u_{x x x x}+\rho u_{t t}-\rho \Omega^{2} u=0, \quad 0<x<L, \\
u(0, t)=0, \quad u_{x}(0, t)=0, \\
E I u_{x x x}(L, t)=\alpha_{1} f_{1}(t), \quad-E I u_{x x}(L, t)=\alpha_{2} f_{2}(t),
\end{gathered}
$$

where a subscript denotes the partial differential with respect to the corresponding variable. In equations (1)-(3), EI and $\rho$ are the flexural rigidity and the mass per unit length of the beam, respectively, which are constants by assumption, $\Omega$ is the constant angular velocity of the rigid body, and $L$ is the length of the beam. Equation (1) is the balance of forces at $x$ along the vertical axis, equation (2) gives the boundary conditions at the clamped end, equation (3) is the boundary conditions at the free end, $f_{1}(t)$ and $f_{2}(t)$ are the boundary control force and the boundary control moment applied to the free end of the beam, respectively, and $\alpha_{1}$ and $\alpha_{2}$ are either 0 or 1 . Note that, for $i=1,2, \alpha_{i}=0$ means that the corresponding boundary control $f_{i}(t)$ is not applied.

We note that the rigid body angular momentum equation is omitted here. We assume that appropriate forces and torques applied to the rigid body to maintain the constant angular velocity $\Omega$ and to keep the center of mass $O$ fixed. For more details of this model, as well as for some control applications, see Morgül (1991).

The problems we want to solve are:

(i) Find control laws for the boundary controls $f_{1}(t)$ and $f_{2}(t)$ so that, for $|\Omega|$ sufficiently small, the solutions of 
equations (1)-(3) satisfy the following asymptotic relations: for all $0 \leq x \leq L$

$$
\lim _{t \rightarrow \infty} u(x, t)=0, \quad \lim _{t \rightarrow \infty} u_{t}(x, t)=0 .
$$

(ii) Find relations between the constant angular velocity $\Omega$ and the stability of the solutions of equations (1)-(3).

To stabilize the system given by equations (1)-(3), for $i=1,2$ when $\alpha_{i} \neq 0$, we propose the following feedback control law:

$$
\begin{gathered}
\dot{w}_{i}=A_{i} w_{i}+b_{i} r_{i}(t), \\
f_{i}(t)=c_{i}^{r_{w}} w_{i}+d_{i} r_{i}(t)+k_{i} \int_{-\infty}^{t} r_{i}(\tau) \mathrm{d} \tau
\end{gathered}
$$

where, for $i=1,2, w_{i} \in \mathbf{R}^{n_{i}}$ is the actuator state, $A_{i} \in \mathbf{R}^{n_{i} \times n_{i}}$ is a constant matrix, $b_{i}, c_{i} \in \mathbf{R}^{n_{i}}$ are constant column vectors, $d_{i}$ and $k_{i}$ are constant real numbers, and the superscript $T$ stands for transpose. Note that when $\alpha_{i}=0$, the corresponding boundary condition in equation (3) is homogeneous, hence the corresponding controller given by equations (5)-(6) is absent. As an application of this type of control laws to the stabilization of flexible beams, see Morgül $(1992 a, b)$

The controller input $r_{i}(t)$ is defined as:

$$
r_{1}(t)=u_{t}(L, t), \quad r_{2}(t)=u_{x t}(L, t) .
$$

Note that because of the boundary conditions equation (2) we have:

$$
\int_{-\infty}^{t} r_{1}(\tau) \mathrm{d} \tau=u(L, t), \quad \int_{-\infty}^{t} r_{2}(\tau) \mathrm{d} \tau=u_{x}(L, t) .
$$

When $\alpha_{i} \neq 0$, we assume the following throughout the paper: for $i=1,2$.

Assumption 1. All eigenvalues of $A_{i} \in \mathbf{R}^{n_{i} \times n_{i}}$ have negative real parts.

Assumption 2. $\left(A_{i}, b_{i}\right)$ is controllable and $\left(A_{i}, c_{i}\right)$ is observable.

Assumption 3. $d_{i} \geq 0, \quad k_{i} \geq 0$; moreover there exists a constant $\gamma_{i}$, such that $d_{i} \geq \gamma_{i} \geq 0$, and that the following holds:

$$
d_{i}+\mathscr{R} e\left\{c_{i}^{T}\left(j \omega I-A_{i}\right)^{-1} b_{i}\right\}>\gamma_{i}, \quad \omega \in \mathbf{R} .
$$

If we take the Laplace transform in equations (5) and (6) and use zero initial conditions, we obtain:

$\hat{f}_{i}(s)=\left[d_{i}+c_{i}^{T}\left(s I-A_{i}\right)^{-1} b_{i}+\frac{k_{i}}{s}\right] \hat{r}_{i}(s)=g_{i}(s) \hat{r}_{i}(s)$

$$
i=1,2,
$$

where a hat denotes the Laplace transform of the corresponding variable. This, together with equation (9) implies that the transfer function in equation (10) is a strictly positive real function.

\section{Stability results}

3.1 Existence, uniqueness and stability. Let the Assumptions (1-3), or equivalently the Assumptions (i-ii) stated in the previous section hold. Then, for $i=1,2$ it follows from the Kalman-Yakubovich Lemma that given any symmetric positive definite matrix $Q_{i} \in \mathbf{R}^{n_{i} \times n_{i}}$, there exists a symmetric positive definite matrix $P_{i} \in \mathbf{R}^{n_{i} \times n_{i}}$ and a vector $q_{i} \in \mathbf{R}^{n_{i}}$ satisfying:

$$
\begin{gathered}
A_{i}^{T} P_{i}+P_{i} A_{i}=-q_{i} q_{i}^{T}-\epsilon_{i} Q_{i}, \\
P_{i} b_{i}-\frac{1}{2} c_{i}=\sqrt{d_{i}-\gamma_{i}} q_{i}
\end{gathered}
$$

provided that $\epsilon_{i}>0$ is sufficiently small, see Vidyasagar (1978, p. 201).

To analyze the system given by equations (1)-(3), (5), (6), which will be referred to as the system $\mathscr{P}$, we first define the function space $\mathscr{H}$ as follows:

$$
\mathscr{H}:=\left\{\left(\begin{array}{llll}
u & v & w_{1} & w_{2}
\end{array}\right)^{T} \mid u \in \mathbf{H}_{0}^{2}, v \in \mathbf{L}^{2}, w_{1} \in \mathbf{R}^{n_{1}}, w_{2} \in \mathbf{R}^{n_{2}}\right\},
$$

where the spaces $\mathbf{L}^{2}$ and $\mathbf{H}_{0}^{k}$ are defined as follows:

$$
\begin{gathered}
\mathbf{L}^{2}=\left\{f:[0, L]-\mathbf{R} \mid \int_{0}^{L} f^{2} \mathrm{~d} x<\infty\right\}, \\
\mathbf{H}_{0}^{k}=\left\{f \in \mathbf{L}^{2} \mid f, f^{\prime}, f^{\prime \prime}, \ldots, f^{(k)} \in \mathbf{L}^{2}, f(0)=f^{\prime}(0)=0\right\} .
\end{gathered}
$$

The equations of the system $\mathscr{S}$ can be written in the following abstract form:

$$
\dot{z}=A z, \quad z(0) \in \mathscr{H},
$$

where $z=\left(u \quad u, w_{1} w_{2}\right)^{T} \in \mathscr{H}$, the operator $A: \mathscr{H} \rightarrow \mathscr{H}$ is a linear unbounded operator defined as

$$
A\left(\begin{array}{c}
u \\
v \\
w_{1} \\
w_{2}
\end{array}\right)=\left[\begin{array}{c}
v \\
\Omega^{2} u-\frac{E I}{\rho} u_{x x x x} \\
A_{1} w_{1}+b_{1} v(L) \\
A_{2} w_{2}+b_{2} v_{x}(L)
\end{array}\right]
$$

The domain $D(A)$ of the operator $A$ is defined as:

$$
\begin{aligned}
D(A)= & \left\{\left(u v w_{1} w_{2}\right)^{T} \mid u \in \mathbf{H}_{0}^{4}, v \in \mathbf{H}_{0}^{2}, w_{1} \in \mathbf{R}^{n_{1}}, w_{2} \in \mathbf{R}^{n_{2}} ;\right. \\
& -E I u_{x x x}(L)+\alpha_{1}\left[c_{1}^{T} w_{1}+d_{1} v(L)+k_{1} u(L)\right]=0, \\
& \left.E I u_{x x}(L)+\alpha_{2}\left[c_{2}^{T} w_{2}+d_{2} v_{x}(L)+k_{2} u_{x}(L)\right]=0\right\} .
\end{aligned}
$$

Remark 1. We note that when $\alpha_{i}=0$ for $i=1$ or $i=2$, the terms corresponding to $w_{i}$ in equations (13) and (18) and the corresponding row in equation (17) will be absent.

Let the Assumptions (1-3) hold, for $i=1,2$ let $Q_{i} \in \mathbf{R}^{n_{i} \times n_{i}}$ be an arbitrary symmetric positive definite matrix and let $P_{i} \in \mathbf{R}^{n_{i} \times n_{i}}, q_{i} \in \mathbf{R}^{n_{i}}$ be the solutions of equations (11) and (12) where $P_{i}$ is also a symmetric and positive definite matrix. In $\mathscr{K}$ we define the following 'energy' function:

$$
\begin{aligned}
E(t)=\frac{1}{2} \int_{0}^{L} \rho u_{i}^{2} \mathrm{~d} x & +\frac{1}{2} \int_{0}^{L} E l u_{x x}^{2} \mathrm{~d} x-\frac{1}{2} \rho \Omega^{2} \int_{0}^{L} u^{2} \mathrm{~d} x \\
& +\frac{1}{2} \sum_{i=1}^{2} \alpha_{i} k_{i}\left(\int_{-\infty}^{t} r_{i}(\tau) \mathrm{d} \tau\right)^{2}+\sum_{i=1}^{2} \alpha_{i} w_{i}^{T} P_{i} w_{i} .
\end{aligned}
$$

In the sequel, we need the following inequalities:

$$
\begin{gathered}
u^{2}(s, t) \leq L \int_{0}^{L} u_{x}^{2} \mathrm{~d} x \leq L^{2} \int_{0}^{L} u_{x x}^{2} \mathrm{~d} x, \\
u_{x}^{2}(s, t) \leq L \int_{0}^{L} u_{x x}^{2} \mathrm{~d} x \quad s \in[0, L],
\end{gathered}
$$

where equation (20) follows from boundary conditions equation (2) and Jensen's inequality, Royden (1968, p. 110). Assume that $\Omega$ is sufficiently small, so that $E I>2 \Omega^{2} L^{3}$. Then,

(1) It follows from equation (20) that $E(t)$ given by equation (19) is always positive.

(2) $E(t)=0$ for $t \in[a, b]$ iff $u(x, t)=0, u_{t}(x, t)=0$ almost everywhere on $[0, L]$ for all $t \in[a, b]$ and $w(t)=0$ for $t \in[a, b]$. This follows from equations (19) and (2).

(3) One can define an 'energy' inner product in $\mathscr{H}$ for which the energy $E(t)$ given by equation (19) becomes a norm on $\mathscr{H}$. Also, with this inner product, $\mathscr{H}$ becomes a Hilbert space.

Lemma 1 . Consider the system given by equation (16). Let the Assumptions (1-3) hold. If $\alpha_{1}+\alpha_{2}>0$ then, for $|\Omega|$ sufficiently small, we have:

(i) the operator $A$ generates a $C_{0}$ semigroup $T(t)$ in $\mathscr{t}$, moreover if $z(0) \in D(A)$, then $z(t)=T(t) z(0), t \geq 0$, is the unique classical solution of equation (16) and $z(t) \in D(A)$, 
$t \geq 0$ (for the terminology on semigroup theory, the reader is referred to Pazy (1983))

(ii) the energy $E(t)$ given by equation (19) asymptotically decays to zero along the classical solutions of equation (16).

Remark 2. Note that the condition $\alpha_{1}+\alpha_{2}>0$ means that at least one of the boundary controllers given by equations (3), (5) and (6) is applied.

Proof. (i) By using equation (19), we obtain:

$$
\begin{aligned}
\frac{\mathrm{d} E}{\mathrm{~d} t}= & -E I \int_{0}^{L} u_{x x x x} u_{t} \mathrm{~d} t+E I \int_{0}^{L} u_{x x} u_{x x t} \mathrm{~d} t \\
& +\sum_{i=1}^{2} \alpha_{i} k_{i} r_{i}(t) \int_{-\infty}^{t} r_{i}(\tau) \mathrm{d} \tau \\
& +\sum_{i=1}^{2} \alpha_{i} w_{i}^{T}\left(A_{i}^{T} P_{i}+P_{i} A_{i}\right) w_{i} \\
& +2 \sum_{i=1}^{i=2} \alpha_{i} w_{i}^{T} P_{i} b_{i} r_{i}(t) \\
= & -\sum_{i=1}^{2} \alpha_{i} f_{i}(t) r_{i}(t)-\sum_{i=1}^{2} \alpha_{i} \epsilon_{i} w_{i}^{T} Q_{i} w_{i} \\
& +\sum_{i=1}^{2} \alpha_{i} k_{i} r_{i}(t) \int_{-\infty}^{t} r_{i}(\tau) \mathrm{d} \tau+2 \sum_{u=1}^{i=2} \alpha_{i} w_{i}^{T} P_{i} b_{i} r_{i}(t) \\
= & -\sum_{i=1}^{2} \alpha_{i} \gamma_{i} r_{i}^{2}(t)-\sum_{i=1}^{2} \alpha_{i} \epsilon_{i} w_{i}^{T} Q_{i} w_{i} \\
& -\sum_{i=1}^{2} \alpha_{i}\left[\sqrt{d_{i}-\gamma_{i}} r_{i}(t)-w_{i}^{T} q_{i}\right]^{2},
\end{aligned}
$$

where to obtain the first equation, we differentiated equation (19) with respect to time, used equation (5); to obtain the second equation we integrated by parts twice, used equations (2), (3) and (11); and to obtain the last equation we used equations (6) and (12). Since $\dot{E} \leq 0$, it follows that $E(t)$ is a nonincreasing function of time along the solutions of the system $\mathscr{S}$. From equation (21) it follows that $A$ is dissipative on $\mathscr{H}$. It can also be shown that the operator $\lambda I-A: \mathscr{H} \rightarrow \mathscr{H}$ is onto for all $\lambda>0$ (see Morgül, 1991 for similar proofs). Therefore, it follows that $D(A)$ is dense in $\mathscr{H}$ (see Pazy, 1983 , p. 16). Hence from Lumer-Phillips theorem, Pazy (1983, p. 14), we conclude that $A$ generates a $C_{0}$ semigroup $T(t)$ on $\mathscr{H}$. That $z(t)=T(t) z(0), t \geq 0$ is the unique classical solution of equations (1)-(4), equations (7)-(9) when $z(0) \in D(A)$, and that $z(t) \in D(A), t \geq 0$ follows from the semigroup property (see Pazy, 1983).

(ii) It can be shown that the operator $(\lambda I-A)^{-1}: \mathscr{H} \rightarrow \mathscr{H}$ is compact for $\lambda \geq 0$ (see Morgül, 1991 for similar proofs). This implies that the spectrum of the operator $A$ consists entirely of countably many) isolated eigenvalues (see Kato, 1980 , p. 187). Since by equation (21) the classical solutions of equation (16) are bounded in $\mathscr{K}$, it follows from the standard results in the semigroup theory that the trajectories corresponding to the classical solutions of equation (16) are precompact in $\mathscr{H}$ (see e.g. Saperstone, 1981, p. 241). Therefore, from LaSalle's invariance principle (see Saperstone, 1981, p. 78), it follows that the trajectories of equation (16) asymptotically approach the maximal invariant set $\mathscr{C} \subset \mathscr{H}$, where $\mathscr{C}$ is defined as:

$$
\mathscr{C}:=\left\{\left(\begin{array}{llll}
u & u & w_{1} & w_{2}
\end{array}\right)^{T} \in \mathscr{H} \mid \mathrm{d} E / \mathrm{d} t=0\right\}
$$

and $d E / d t$ is given by equation (21).

We claim that, for $|\Omega|$ sufficiently small, the only possible classical solution of equation (16) which lies in $\mathscr{C}$ is the zero solution (later we show that $|\Omega|$ should be smaller than the first critical frequency of the uncontrolled, nonrotating beam).

From equation (21), it follows that the solutions of equation (16), which lie in $\mathscr{C}$ must satisfy the following: for $i=1,2$

$$
\begin{gathered}
\alpha_{i} w_{i}(t)=0, \quad \alpha_{i} r_{i}(t)=0 \quad t \geq 0, \\
E I u_{x x x}(L, t)=\alpha_{1} k_{1} u(L, t), \quad-E I u_{x x}(L, t)=\alpha_{2} k_{2} u_{x}(L, t) .
\end{gathered}
$$

Hence, to find the solutions of equation (16) which lie in $\mathscr{C}$, we must solve equations (1), (2), (22) and (23). Since all of the boundary conditions are separable, to find such a solution, we use the method of separation of variables, that is we express the solutions of equation (1) as follows:

$$
u(x, t)=Y(x) B(t) \quad 0 \leq x \leq L, \quad t \geq 0,
$$

where $Y:[0, L] \rightarrow \mathbf{R}$ and $B: \mathbf{R}_{+} \rightarrow \mathbf{R}$ are smooth functions. In the sequel, we suppress the dependency of $Y$ and $B$ on $x$ and $t$, and write $u=Y B$.

Using equation (24) in (1) and then dividing by $Y B$, we obtain:

$$
E I \frac{Y^{\prime \prime \prime \prime}}{Y}+\rho \frac{\ddot{B}}{B}-\rho \Omega^{2}=0 \quad 0 \leq x \leq L \quad t \geq 0 .
$$

Since $Y$ depends only on $x$ and $B$ depends only on $t$, we mus have the following for some $\lambda \in \mathbf{R}$ :

$$
\begin{gathered}
\frac{\ddot{B}}{B}=-\lambda^{2} \quad t \geq 0, \\
E I \frac{Y^{\prime \prime \prime}}{Y}=\tau^{4} \quad \tau^{4}=\frac{\rho}{E I}\left(\lambda^{2}+\Omega^{2}\right) \quad 0 \leq x \leq L .
\end{gathered}
$$

Using equation (24) in (2) and equation (23), we conclude that in order to have a nontrivial solution $Y(\cdot)$ must satisfy the following boundary conditions:

$$
\begin{gathered}
Y(0)=0, \quad Y^{\prime}(0)=0, \\
E I Y^{\prime \prime \prime}(L)=\alpha_{1} k_{1} Y(L), \quad-E I Y^{\prime \prime}(L)=\alpha_{2} k_{2} Y^{\prime}(L) .
\end{gathered}
$$

We consider the following cases

Case 1. $\lambda=0, \boldsymbol{\Omega}=0$. In this case, it can easily be shown that the solution of equation (27) which satisfies equations (28) and (29) is the zero solution, which implies that $u(x, t)=0$, for $0 \leq x \leq L, t \geq 0$.

Case 2. $\lambda=0, \Omega \neq 0$. In this case, the solution of equation (27) which satisfies equation (28) is given as follows:

$$
Y(x)=c_{1}(\cosh \tau L-\cos \tau L)+c_{2}(\sinh \tau L-\sin \tau L)
$$

$$
0 \leq x \leq L \text {, }
$$

where $\cosh (\cdot)$ and $\sinh (\cdot)$ are hyperbolic cosine and hyperbolic sine functions, respectively, $c_{1}$ and $c_{2}$ are constants to be determined from equation (29). Using equation (30) in (29), we conclude that in order to have a nontrivial solution, $\tau$ must satisfy the following:

$$
\begin{aligned}
& \quad(E I)^{2} \tau^{5}(1+\cosh y \cos y)+E I \alpha_{2} k_{2} \tau^{4} \\
& \times(\sinh y \cos y+\cosh y \sin y) \\
& +E I \alpha_{1} k_{1} \tau^{2}(\cosh y \sin y-\sinh y \cos y) \\
& +\alpha_{1} \alpha_{2} k_{1} k_{2} \tau(1-\cosh y \cos y)=0,
\end{aligned}
$$

where $y=\tau L$. Since the spectrum of the system $\mathscr{S}$ consists of countably many isolated eigenvalues, it can be shown that equation (31) has countably many solutions $\tau>0$. Let $\tau_{1}>0$ be the smallest of such solutions (e.g. when $\alpha_{2}=0$, $1.875 \leq \tau_{1} L \leq 3.927$, and when $\alpha_{1}=0,1.875 \leq \tau_{1} L \leq 2.365$ ). Then, it is clear that for $|\Omega|<\sqrt{E I / \rho} \tau_{1}^{2}$, equation (31) does not have a solution, hence we must have $c_{1}=c_{2}=0$ in equation (30), and consequently the only possible solution is the zero solution (see equations (30) and (24)).

Case 3. $\lambda \neq 0$. In this case, it follows from equation (22) that we have $Y(L)=0$, or $Y^{\prime}(L)=0$, depending on $\alpha_{1}=1$ or $\alpha_{2}=1$, respectively; for otherwise we must have $\dot{B}(t) \equiv 0$, which contradicts with equation (26). It can be easily shown that there does not exist a $\tau>0$ given by equation (27) such that equation (30) satisfy both equation (29) and the boundary conditions stated above. Hence we must have $c_{1}=c_{2}=0$ in equation (30), and consequently the only possible solution is the zero solution (see equations (30) and (24)).

From the Cases 1,2 and 3, we conclude that the only possible solution which lie in $\mathscr{C}$ is the zero solution. Hence from LaSalle's invariance principle, both the energy $E(t)$ and 
the solutions $z(t)$ of equation (16) asymptotically decay to zero.

Remark 3. Let $\alpha_{i}=1$ for $i=1,2$, and consider the corresponding controller transfer function given by equation (10). The conclusions of the Lemma 1 still hold if $d_{i}=0$, in which case the corresponding transfer function becomes strictly proper. Hence the class of stabilizing controllers given by equations (5) and (6) include some strictly proper controllers, which is important for practical reasons since physical devices show strictly proper behaviour, see Horowitz (1963). $\square$

In the sequel, first we show that in certain cases the decay of the energy is in fact exponential. Later we obtain some bounds on $\Omega$ to ensure stability.

Theorem 1. Consider the system $\mathscr{Y}$. Assume that $\alpha_{1}=1$ and that $\gamma_{1}>0$ (see equation (9)). Let the Assumptions (1-3) hold. Then for $k_{1}$ and $k_{2}$ sufficiently small, there exists an angular velocity $\Omega_{c r}$ such that, for all $\Omega,|\Omega|<\Omega_{c r}$, the energy given by equation (19) decays exponentially to zero along the solutions of the system $\mathscr{S}$.

Proof. First, we assume that $|\Omega|$ is sufficiently small so that the energy given by equation (19) is non-negative. We define the following function $V(t)$ :

$$
V(t)=2(1-\epsilon) t E(t)+2 \int_{0}^{L} \rho x u_{r} u_{x} \mathrm{~d} x
$$

where $\epsilon \epsilon(0,1)$ is an arbitrary constant.

In the sequel, we need the following inequality:

$$
a b \leq \delta^{2} a^{2}+b^{2} / \delta^{2}, \quad a, b, \delta \in \mathbf{R}, \quad \delta \neq 0 .
$$

It follows from equations (32), (19), (20) and (33) that there exists a constant $C>0$ such that: for $t \geq 0$

$$
[2(1-\epsilon) t-C] E(t) \leq V(t) \leq[2(1-\epsilon) t+C] E(t) .
$$

Differentiating equation (32) with respect to time, using integration by parts, equation (33), the boundary conditions (2), (3) and (20), we obtain:

$$
\begin{aligned}
\dot{V}(t)= & 2(1-\varepsilon) E(t)+2(1-\varepsilon) t \dot{E}(t)-2 E I \\
& \times \int_{0}^{L} x u_{x x x x} u_{x} \mathrm{~d} x+2 \int_{0}^{L} \rho x u_{t} u_{x t} \mathrm{~d} x+2 \rho \Omega^{2} \int_{0}^{L} x u u_{x} \mathrm{~d} x \\
\leq & -\varepsilon \int_{0}^{L} \rho u_{t}^{2} \mathrm{~d} x-\left[2(1-\varepsilon) t \epsilon_{1} w_{1}^{T} Q_{1} w_{1}\right. \\
& \left.-2(1-\varepsilon) w_{1}^{T} P_{1} w_{1}-\left(\frac{4 L}{\delta_{1}^{2}}\right)\left(c_{1}^{T} w_{1}\right)^{2}\right] \\
& -\left[2(1-\varepsilon) t \gamma_{1}-\frac{4 L d_{1}^{2}}{\delta_{1}^{2}}-\rho L\right] u_{t}^{2}(L, t) \\
& -2(1-\varepsilon) t \sum_{i=1}^{2}\left[\sqrt{d_{i}-\gamma_{i}} r_{i}(t)-w_{i}^{T} q_{i}\right]^{2} \\
& -\alpha_{2}\left[2(1-\varepsilon) t \gamma_{2}-\frac{2 d_{2}}{\delta_{3}^{2}}-\frac{4 d_{2}^{2} L}{E I}\right] u_{x i}^{2}(L, t) \\
& -\alpha_{2}\left[2(1-\varepsilon) t \epsilon_{2} w_{2}^{T} Q_{2} w_{2}\right. \\
& \left.-2(1-\varepsilon) w_{2}^{T} P_{2} w_{2}-\left(\frac{2}{\delta_{2}^{2}}+\frac{4 L}{E I}\right)\left(c_{2}^{T} w_{2}\right)^{2}\right] \\
& -\left[(\varepsilon+2) E I-2 L \delta_{1}^{2}-\frac{4 L^{3} k_{1}^{2}}{\delta_{1}^{2}}-2 \alpha_{2} L \delta_{2}^{2}\right. \\
& \left.-2 \alpha_{2} d_{2} \delta_{3}^{2} L-\frac{4 k_{2}^{2} L^{2}}{E I}-\rho \Omega^{2} L^{3}(1+L)\right] \int_{0}^{L} u_{x x}^{2} \mathrm{~d} x .
\end{aligned}
$$

where $\delta_{1} \neq 0, \delta_{2} \neq 0, \delta_{3} \neq 0$ are arbitrary constants.

By choosing $\delta_{1}, \delta_{2}, \delta_{3}$ and then $k_{1}, k_{2}$ and $|\Omega|$ sufficiently small, the last line in equation (35) can be made negative. Hence, from equation (35), it follows that there exists a $T \geq 0$ such that the following holds

$$
\dot{V}(t) \leq 0, \quad t \geq T .
$$

This, together with equation (34) implies that

$$
E(t) \leq \frac{V(T)}{(2(1-\varepsilon) t-C}, \quad t \geq T .
$$

By equation (21), $E(t) \leq E(0)$, hence equation (34) implies that $V(T)<\infty$. Let $\Omega_{c r}$ be the maximum number for which $E(t) \geq 0$ and equation (36) holds. Then, from equations (21) and (37) we conclude that for $|\Omega|<\Omega_{c r}$, the following holds:

$$
\int_{0}^{\infty} E^{2}(t) \mathrm{d} t<\infty
$$

Exponential decay follows from a result due to Pazy (see Pazy, 1983, p. 116), that is there exists positive constants $M$, $\delta$ such that the following holds

$$
E(t) \leq M e^{-i t}, \quad t \geq 0 .
$$

Remark 4. Although in the Theorem 1 we only prove that the exponential decay of the energy holds for $k_{1}$ and $k_{2}$ sufficiently small, we conjecture that the same result holds for arbitrary positive $k_{1}$ and $k_{2}$. Main motivation for this conjecture stems from the asymptotic decay of energy (see Lemma 1) and from the proof of the Theorem 3 in the work of Chen (1979).

(ii) Stability bound for $\Omega$. For simplicity in the subsequent calculations, we assume that $\alpha_{1}=1, d_{1}>0$ and that $\alpha_{2}=0$. As stated in the Lemma 1 , the spectrum of $A$ given by equation (17) consists entirely of isolated eigenvalues with finite multiplicities. By the Theorem 1 , the energy $E(t)$ decays exponentially to zero for small $\Omega$, hence all eigenvalues must be in the open left half of the complex plane. Since these eigenvalues depend continuously on $\Omega$ (see equations (27) and (31)), as we increase $|\Omega|$ these eigenvalues change continuously. Hence, if we can find the least value $\boldsymbol{\Omega}_{c r}$ for $\boldsymbol{\Omega}$ for which at least one eigenvalue of $A$ crosses the imaginary axis, then because of continuity we can say that for $|\Omega|<\Omega_{c r}$ all eigenvalues of $A$ have negative real parts. In the sequel we will find such a bound.

Taking the Laplace transform of equations (1)-(3), replacing equations (5) and (6) by (10), we obtain the following equation which gives the eigenvalues of the system $\mathscr{Y}:$

$$
\beta^{3} X(\beta)-\left(s g_{1}(s) / E I\right) Y(\beta)=0, \quad \beta^{4}=-(\rho / E I)\left(s^{2}-\Omega^{2}\right),
$$

where $X(\cdot)$ and $Y(\cdot)$ are given by the following:

$$
X(\tau)=1+\cosh \tau L \cos \tau L,
$$

$Y(\tau)=\sinh \tau L \cos \tau L-\cosh \tau L \sin \tau L$.

Putting $s=j \omega$ in equation (40), we obtain:

$$
\beta=a e^{j 2 n \pi / 4}, \quad n=0,1,2,3,
$$

where $a=\sqrt[4]{\rho / E I\left(\omega^{2}+\Omega^{2}\right)}$

Putting $\beta=a$ (other choices of $n$ in equation (42) will yield the same result) and $g_{1}(j \omega)=R(\omega)+j l(\omega)$ in equation (40), we obtain:

$$
\left[a^{3} X(a)+(\omega I(\omega) / E I) Y(a)\right]-j(\omega R(\omega) / E I) Y(a)=0 .
$$

By equation (9), $R(\omega)>0$ for all $\omega \in \mathbf{R}$, and by equation (10) $R(\omega)$ does not have any singularity at $\omega=0$. Hence, from equation (43) we conclude that $\omega Y(a)=0$. If $Y(a)=0$, equation (43) implies that $a^{3} X(a)=0$; but since $a \neq 0$ (see equation (42)), and since $X(a)$ and $Y(a)$ do not have any common zero, this is impossible. Hence the only relevant case is $\omega=0$; that is the root loci of the eigenvalues of the system $\mathscr{S}$ with respect to the parameter $\Omega$ may cross the imaginary axis only at the origin.

Let $\alpha_{1}$ be the smallest positive real number satisfying $X\left(\alpha_{1}\right)=0 \quad\left(\alpha_{1} L \simeq 1.875\right)$, and let, for $n \geq 2, \quad \alpha_{n}$ be the smallest positive real number satisfying both $X\left(\alpha_{n}\right)=0$ and $\alpha_{n}>\alpha_{n-1}$. Let $\omega_{n C F}$ be defined as $\omega_{n C F}=\alpha_{n}^{2} \sqrt{E I / \rho}$, which is the $n$th critical frequency of a clamped-free beam. Let $\beta_{1}$ 
be the smallest positive real number satisfying $Y\left(\beta_{1}\right)=0$ $\left(\beta_{1} L \simeq 3.927\right.$ ), and let, for $n \geq 2, \beta_{n}$ be the smallest positive real number satisfying both $Y\left(\beta_{n}\right)=0$ and $\beta_{n}>\beta_{n-1}$. Let $\omega_{n C H}$ be defined as $\omega_{n C H}=\beta_{n}^{2} \sqrt{E I / \rho}$, which is the $n$th critical frequency of a clamped-hinged beam.

Fact 1 . Consider the equations given by equations (40) and (43), where $g_{1}(s)$ is given by equation (10). We have the following:

(i) If $k_{1}=0$, then for $|\Omega|<\omega_{1 C F}$, all solutions of equation (43) have negative real parts.

(ii) If $k_{1}>0$, then there exists a $\Omega_{c r}, \omega_{1 C F}<\Omega_{c r}<\omega_{1 C H}$ such that for $|\Omega|<\Omega_{c r}$, all solutions of equation (43) have negative real parts.

Proof.

(i) In this case, $I(\omega)$ does not have any singularity at $\omega=0$, and consequently we have $X(a)=0$. Therefore we conclude that for $|\Omega|<\omega_{1 C F}$, all eigenvalues of the system $\mathscr{S}$, which are the solutions of equations (40), have negative real parts.

(ii) Let $k_{1}>0$ be a given fixed number. Noting that the only relevant case is $\omega=0$, equation (43) can be written as:

$$
\frac{X(a)}{Y(a)}=\frac{k_{1}}{E l a^{3}} \text {. }
$$

The left-hand side of equation (44) is an increasing function of $a$, with zeroes at $\alpha_{n}$ and with discontinuities at $\beta_{n}, n \geq 1$. Also we have $\alpha_{n}<\beta_{n}<\alpha_{n+1}$, see Hurty and Rubinstein (1964). Since the right-hand side of equation (44) is a decreasing function of $a$ and $k_{1}>0$, there exist countably many solutions to equation (44). Let $\gamma_{1}$ be the smallest positive number which solves equation (44) and let $\gamma_{n}$ be the smallest positive number satisfying both equation (44) and $\gamma_{n}>\gamma_{n-1}$. Obviously we have $\alpha_{1}<\gamma_{1}<\beta_{1}$. Let $\omega_{c r}$ be defined as $\omega_{c r}=\gamma_{1}^{2} \sqrt{E I / \rho}$. It follows then that for $|\Omega|<\omega_{c r}$, all solutions of equation (44), hence equation (43), have negative real part.

Moreover, as in Baillieul and Levi (1987), we have the following:

Fact 2 . Consider the equations given by equations $(40)$ and (43), where $g_{1}(s)$ is given by equation $(10)$. We have the following:

(i) If $k_{1}=0$, then for $\omega_{n C F}<|\Omega|<\omega_{(n+1) C F}$, equation

(40) has at least $n$ positive solutions.

(ii) If $k_{1}>0$, then for $\omega_{n C H}<|\Omega|<\omega_{(n+1) C F}$, equation (40) has at least $n$ solutions with positive real parts.

Proof. In these cases we seek positive solutions of equation $(40)$, (i.e. $s>0$ ). From equation (40) we obtain:

$$
\frac{X(\beta)}{Y(\beta)}=\frac{s g_{1}(s)}{E I \beta^{3}},
$$

where $\beta$ is as given in equation (40). Note that $s$ ceases to exist as a real number when $\beta^{2}>\sqrt{E I / \rho} \Omega$ and that $s g_{1}(s)=k_{1}$ when $\beta^{2}=\sqrt{E I / \rho} \Omega$. The right-hand side of equation (45) is a decreasing function of $\beta$ whereas the left-hand side is an increasing function of $\beta$, see the Fact 1 . Then the conclusions of the cases (i) and (ii) can be easily shown by using simple graphs.

Remark 5. Fact 1 gives us an upper bound for $\Omega$ below which all solutions of equation (40), hence all eigenvalues of the operator $A$ have negative real parts. From a mathematical point of view, this information on the eigenvalues is not enough to conclude the stability of the solutions, since the system in question is an infinite dimensional system, see e.g. Pazy (1983). Nevertheless from the Fact 2 we can safely conclude that for $\Omega$ greater than these bounds, the system is unstable, and from an engineering point of view, one expects that the system remains stable for $\Omega$ smaller than these bounds. Note that this engineering intuition can be mathematically justified if an appropriate damping is present in the system.

Remark 6. In the Theorem 1 we show that when $|\Omega|$ sufficiently small, the rate of decay of the associated energy is exponential, i.e. equation (39) holds. This result still holds in the static feedback case (i.e. when $c_{i}=0, i=1,2$ in equation (6)), provided that $d_{1}>0, d_{2} \geq 0$. This can easily be shown by using the Lemma 1 and the Theorem 1 with appropriate modifications. An estimate of the decay rate (i.e. $\delta$ in equation (39)) is not obtained, due to the difficulties in estimating this quantity. Note that, from an engineering point of view, one expects that $\delta$ is equal to the minimum of the real part of all eigenvalues. However, this equality is not always true unless some conditions hold, e.g. if the eigenvectors form a Riezs basis in $\mathscr{H}$. We note that such an estimate, for the static case with nonlinear boundary feedback is obtained in Conrad and Pierre (1990).

\section{Conclusion}

In this paper, we studied the motion of a flexible beam clamped to a rigid base at one end and free at the other end and we assumed that the rigid base is rotating with constant angular velocity. We have shown that, by applying suitable boundary control force and torque at the free end of the beam, one can stabilize the beam vibrations provided that the constant angular velocity of the rigid base is sufficiently small. The class of stabilizing controllers proposed here contains some strictly proper controllers, in which case the closed-loop system is asymptotically stable. If the stabilizing controller generating boundary control force is proper but not strictly proper, then we proved exponential stability. We also have shown that, when position feedback is not used (i.e. $k_{1}=0$ ), the critical angular velocity below which al eigenvalues of the system have negative real part is equal to the $\omega_{1 C F}$, the first critical frequency of the clamped-free beam, and that when position feedback is used (i.e. $k_{1}>0$ ), depending on $k_{1}$, the critical angular velocity can be placed between $\omega_{1 C F}$ and $\omega_{1 C H}$, the first critical frequency of the clamped-free beam and the clamped-hinged beam, respectively. Since $\omega_{\mathrm{ICH}}$ is approximately four times greater than $\omega_{1 C F}$, with position feedback it is possible to rotate the system faster without endangering stability.

An interesting point is, the dynamic boundary control presented in this paper can be used to change the spectrum of the uncontrolled beam (see equation (40)). This change in the spectrum, although limited, can be exploited for some control applications, such as eigenvalue assignment, disturbance rejection, etc.

\section{References}

Baillieul, J. and M. Levi (1987). Rotational elastic dynamics. Physica D, 27D, 43-62.

Chen, G. (1979). Energy decay estimates and exact boundary value controllability for the wave equation in a bounded domain. J. Math. Pures. Appl., 58, 249-273.

Chen, G., M. C. Delfour, A. M. Krall and G. Payre (1987) Modelling, stabilization and control of serially connected beams. SIAM J. of Contr. and Optim., 25, 526-546.

Conrad, F. and M. Pierre (1990). Stabiliztion of EulerBernoulli beam by nonlinear boundary feedback. Research report INRIA, no. 1235, Univ. of Nancy 1, Dept. of Math., France.

Horowitz, I. M. (1963). Synthesis of Feedback Systems. Academic Press, NY.

Hurty, W. C. and M. F. Rubinstein (1964). Dynamics of Structures. Prentice-Hall, Englewood Cliffs, NJ.

Kato, T. (1980). Perturbation Theory for Linear Operators. Springer Verlag, NY.

Morgül, Ö. (1990). Control and stabilization of a flexible beam attached to a rigid body. International J. of Control, 51, 11-31.

Morgül, Ö. (1991). Orientation and stabilization of a flexible beam attached to a rigid body: planar motion. IEEE Trans. on Auto. Control, 36, 953-963.

Morgül, Ö. (1992a). Dynamic boundary control of a Euler-Bernoulli beam. IEEE Trans. on Auto. Control, 37, 639-642. 
Morgül, Ö. (1992b). On the stabilization of the wave equation. In R. F. Curtain (Ed.), Analysis and Optimization of Systems: State and Frequency Domain Approaches for Infinite Dimensional Systems, Lecture Notes in Control and Information Sciences, Vol. 185, pp. 531-543. Springer-Verlag, NY.

Pazy, A. (1983). Semigroups of Linear Operators and
Applications to Partial Differential Equations. SpringerVerlag, NY.

Royden, H. L. (1968). Real Analysis. MacMillan, NY.

Saperstone, S. (1981). Semidynamical Systems in Infinite Dimensional Spaces. Springer-Verlag, NY.

Vidyasagar, M. (1978). Nonlinear Systems Analysis. Prentice-Hall, Englewood Cliffs, NJ. 